\section{Fernando V Siqueira'}

Luiz Augusto Facchini'

Roberto X Piccini'

Elaine Tomasi'

Elaine Thumé

Denise S Silveira"

Vera Vieira'

Pedro C Hallal'
Programa de Pós-graduação em Epidemiologia. Universidade Federal de Pelotas. Pelotas, RS, Brasil

" Secretaria Municipal da Saúde. Pelotas, RS Brasil

\section{Correspondência | Correspondence:}

Fernando Vinholes Siqueira

Programa de Pós-Graduação em Epidemio-

logia

Universidade Federal de Pelotas

Av. Duque de Caxias, 250

96030-002 Pelotas, RS, Brasil

E-mail:fcvsiqueira@uol.com.br

\section{Prevalência de quedas em idosos e fatores associados}

\section{Prevalence of falls and associated factors in the elderly}

\section{RESUMO}

OBJETIVO: O aumento da expectativa de vida e conseqüente crescimento da população de idosos têm gerado modificações em seu perfil de morbimortalidade. Das doenças crônico-degenerativas, as quedas são agravos prevalentes entre aqueles passíveis de prevenção. O objetivo do estudo foi analisar a prevalência de quedas em idosos e a influência de variáveis a elas associadas.

MÉTODOS: Estudo transversal com amostra composta por 4.003 idosos (65 anos ou mais) cuja coleta de dados foi realizada em 2005. Os idosos residiam na área de abrangência de unidades básicas de saúde de 41 municípios, com mais de 100 mil habitantes, de sete estados do Brasil. Para cálculo do nível de significância dos dados foi usado o teste de Wald para heterogeneidade e tendência linear. A análise ajustada foi realizada por regressão de Poisson, com cálculo de razões de prevalência ajustadas.

RESULTADOS: A prevalência de quedas entre os idosos foi de $34,8 \%$, significativamente maior nas mulheres $(40,1 \%)$. Entre os que sofreram quedas, $12,1 \%$ tiveram fratura como conseqüência. A prevalência de quedas associou-se com idade avançada, sedentarismo, autopercepção de saúde como sendo ruim e maior número de medicações referidas para uso contínuo. Não houve diferença na ocorrência de quedas entre os idosos das diferentes modalidades da atenção (unidade de saúde tradicional e Programa Saúde da Família).

CONCLUSÕES: A prevalência de quedas entre os idosos poderia ser diminuída com o planejamento de ações voltadas às suas necessidades nas unidades de saúde, especialmente em relação aos fatores associados passíveis de prevenção.

DESCRITORES: Idoso. Acidentes por quedas. Fatores de risco. Serviços de saúde para idosos. Estudos transversais. 


\begin{abstract}
OBJECTIVE: Increased life expectancy and consequent growth of the elderly population has resulted in changes in the epidemiological morbidity and mortality profile. In the group of chronic degenerative diseases, falls are a prevailing among those preventable conditions. The objective of the study was to assess prevalence of falls in the elderly and to describe their associated factors.
\end{abstract}

METHODS: Cross-sectional study comprising 4,003 elderly subjects (aged 65 years or more) living in areas covered by primary health care in 41 municipalities with more than 100,000 inhabitants from seven Brazilian states. Wald test for heterogeneity and linear trend were used for the estimation of significance level. Adjusted analysis was conducted using Poisson regression and adjusted prevalence rates were estimated.

RESULTS: The prevalence of falls among the elderly was $34.8 \%$, significantly higher in women (40.1\%) than men. Among those who experienced falls, $12.1 \%$ had fractures as a consequence. The prevalence of falls was directly associated with older age, sedentary lifestyle, self-perception of poor health and more chronic drug use reported. The prevalence of falls was similar in the elderly attending different care services (basic health care units and Family Health Program).

CONCLUSIONS: The prevalence of falls among elderly could be reduced through actions targeting their needs in health care units, especially regarding preventable associated factors.

KEY WORDS: Aged. Accidental falls. Risk factors. Health services for the aged. Cross-sectional studies.

\section{INTRODUÇÃO}

O aumento da expectativa de vida da população é uma realidade entre os diversos grupos populacionais. Esta realidade tem determinado uma modificação no perfil demográfico e de morbimortalidade, resultando em envelhecimento da população e conseqüente aumento proporcional das doenças crônico-degenerativas. ${ }^{6,17}$ Há previsões de que a população de pessoas com 50 anos ou mais de idade irá dobrar no mundo até o ano de $2020 .{ }^{6}$ No Brasil, a expectativa do Instituto Brasileiro de Geografia e Estatística (IBGE) é de que a população com mais de 60 anos de idade seja aproximadamente $11 \%$ da população geral até o ano de 2020 . Todas essas mudanças têm despertado grande interesse na área da saúde pública.

O envelhecimento populacional e o aumento da ocorrência de doenças crônico-degenerativas provocam a necessidade da preparação e adequação dos serviços de saúde, incluindo a formação e capacitação de profissionais para o atendimento desta nova demanda. Nesta perspectiva, as quedas de idosos são atualmente uma das preocupações, pela freqüência e pelas conseqüências em relação à qualidade de vida. A prevenção é importante no sentido de minimizar problemas secundários decorrente de quedas. ${ }^{7}$ Estudos têm mos- trado que é possível diminuir a ocorrência de quedas com cuidados simples como: a) promoção da saúde e prevenção de quedas; b) revisão das medicações; c) modificações nos domicílios; d) promoção da segurança no domicílio; e) promoção da segurança fora do domicílio. Intervenções multidisciplinares podem auxiliar no processo de prevenção de quedas no âmbito populacional. ${ }^{20}$

Um estudo realizado na Turquia mostra que $31,9 \%$ dos idosos caíram pelo menos uma vez no último ano. ${ }^{8} \mathrm{Um}$ estudo realizado na Cataluña mostra que $17,9 \%$ das pessoas acima de 65 anos sofreram pelo menos uma queda nos 12 meses anteriores à entrevista e que essa freqüência aumenta com a idade. ${ }^{21}$ No Brasil, cerca de $30 \%$ dos idosos sofrem quedas ao menos uma vez ao ano. ${ }^{15}$

O presente estudo teve o objetivo de analisar a prevalência de quedas em uma população de idosos e a influência de variáveis a elas associadas.

\section{MÉTODOS}

Foi realizado um estudo de delineamento transversal com amostra de indivíduos idosos, com 65 anos ou 
mais, dos estados do Rio Grande do Sul, Santa Catarina, Alagoas, Pernambuco, Paraíba, Rio Grande do Norte e Piauí, no ano de 2005. ${ }^{16}$ Os idosos residiam na área de abrangência de unidades básicas de saúde (UBS) de 41 municípios com mais de 100 mil habitantes.

Os municípios incluídos no estudo compõem o Lote 2 Sul e Nordeste do Estudo de Linha de Base do Projeto de Expansão e Consolidação da Saúde da Família (PROESF). ${ }^{10,16}$ Uma amostra aleatória de 120 UBS foi sorteada em cada um dos lotes estudados, com distintas modalidades de atenção básica - Programa Saúde da Família (PSF) e tradicional. A partir de listas produzidas pelos municípios, as UBS foram selecionadas na razão de duas UBS do grupo PSF para uma UBS do grupo tradicional. A seleção da amostra nos municípios foi proporcional à capacidade instalada de sua rede básica, pareando as UBS por tamanho da área física. Na região Sul, obteve-se uma amostra de 69 UBS de PSF e 51 tradicionais. No Nordeste, a amostra foi constituída de 79 UBS de PSF e 41 tradicionais. As UBS sorteadas orientaram a seleção da amostra de idosos da área de abrangência dos serviços.

Após a amostragem das UBS, a amostra foi constituída de forma sistemática e independente para os idosos, residentes na área de abrangência das UBS selecionadas. A amostra foi estimada em 2.100 indivíduos por lote (Sul e Nordeste, 4.200 indivíduos no total), de modo a alcançar poder estatístico de no mínimo $80 \%$ para eventos de prevalência aproximada de $25 \%$. O número estimado de indivíduos para cada lote (2.100) foi dividido pelo número total de UBS (120 UBS/lote), obtendo-se a necessidade de entrevistar 18 idosos na área de abrangência de cada uma das UBS. ${ }^{10}$

A estratégia para delimitação da área de abrangência da UBS incluiu a obtenção prévia de seu mapa e uma estimativa populacional a partir das áreas censitárias do IBGE. A localização dos indivíduos na área delimitada ocorreu por meio de amostragem sistemática e incluiu apenas um indivíduo por domicílio. A coleta de dados foi realizada por questionário, aplicado por 15 supervisores de campo após período de treinamento.

Com base no cálculo original de tamanho de amostra, 4.200 indivíduos com 65 anos ou mais seriam localizados nos domicílios da amostra. No entanto, foram identificadas e entrevistadas 4.003 pessoas dessa faixa etária. Com essa amostra, a margem de erro para a estimativa de prevalência de quedas foi de 1,5 pontos percentuais. Para avaliação de associações, o estudo teve poder de $80 \%$ para detectar como significativas razões de prevalência de 1,2 ou maiores, para exposições que afetam de $10 \%$ a $90 \%$ da população, com nível de confiança de $95 \%$.
A definição de queda utilizada foi: "um evento não intencional que tem como resultado a mudança de posição do indivíduo para um nível mais baixo em relação à sua posição inicial". ${ }^{9}$ O questionário utilizado no estudo foi padronizado e pré-testado. A pergunta sobre quedas foi: "No último ano, o(a) Sr.(a) caiu alguma vez?". Para os indivíduos que responderam positivamente, questionouse a ocorrência de fratura resultante da queda e o local anatômico fraturado. As perguntas utilizadas para investigar fraturas e local anatômico foram: a) Em alguma dessas quedas ocorreu uma fratura? Em caso de resposta afirmativa, foi perguntado: b) Qual parte do corpo foi fraturada?.

As variáveis independentes incluídas na análise foram: sexo; faixa etária (65 a 70 anos, 71 a 75 anos, 76 a 80 anos, mais de 80 anos); cor da pele (segundo o entrevistador); situação conjugal; situação ocupacional atual (ativo ou aposentado); escolaridade (maior nível educacional alcançado); tabagismo (nunca fumou, ex-fumante, fumante atual); nível socioeconômico (Classificação da Associação Brasileira de Empresas de Pesquisa*); sedentarismo (escore de atividade física inferior à 150 min por semana, obtido a partir do Questionário Internacional de Atividade Física - IPAQ); ${ }^{5}$ autopercepção de saúde; tipo de UBS que atende o domicílio; número de medicamentos referidos para uso contínuo.

As análises descritivas incluíram cálculos de proporções e respectivos intervalos de confiança de $95 \%$. Na análise bruta, a prevalência de quedas foi calculada para cada grupo das variáveis independentes, e o nível de significância foi testado usando os testes de Wald para heterogeneidade e tendência linear. A análise ajustada foi realizada por regressão de Poisson $^{2}$ com cálculo de razões de prevalência ajustadas, intervalos de confiança de $95 \%$ e valores de significância usando os mesmos testes descritos acima. Todas as análises levaram em consideração o desenho amostral, utilizando um modelo hierárquico de determinação para o desfecho.

O Comitê de Ética da Faculdade de Medicina da Universidade Federal de Pelotas aprovou o protocolo do estudo, e consentimento informado foi obtido de todos os participantes.

\section{RESULTADOS}

A amostra incluiu $61 \%$ de mulheres, $70 \%$ de pessoas de cor da pele branca, $45 \%$ de nível socioeconômico E. Aproximadamente $45 \%$ da amostra foi composta por pessoas viúvas, $50 \%$ nunca freqüentaram a escola e $73 \%$ eram aposentados. A idade média foi de 73,9

\footnotetext{
* Associação Brasileira de Empresas de Pesquisas. Critério de classificação econômica Brasil. Disponível em: http://www.abep.org/codigosguias/ABEP_CCEB.pdf [Acesso em 28 Jun 2007]
} 
anos (variando de 65 a 113 anos); 57,0\% dos indivíduos estudados fumam ou já fumaram, enquanto $57,9 \%$ foram classificados como sedentários e $65 \%$ têm uma percepção ruim ou regular de sua saúde. Em relação a medicamentos referidos para uso contínuo, aproximadamente $70 \%$ da amostra referiu a necessidade de utilização de pelo menos um medicamento continuamente (Tabela 1).

$\mathrm{Na}$ amostra estudada, a prevalência de quedas entre os idosos foi de $34,8 \%$. Entre aqueles que relataram queda durante os 12 meses anteriores à pesquisa, $55 \%$ tiveram uma única queda. Entre os que experimentaram queda, $12,1 \%$ tiveram fratura como conseqüência da queda: $46 \%$ nos membros superiores, $28 \%$ nos membros inferiores, $11 \%$ no tronco, $5,5 \%$ na face e os valores restantes distribuídos entre outros locais. Em relação aos membros superiores, as fraturas de braço foram as mais prevalentes $(26,2 \%)$, seguido das de pulso $(8,5 \%)$ e mão $(5,5 \%)$. Nos membros inferiores, as maiores prevalências foram para os pés $(7,9 \%)$, fêmur $(6,7 \%) \mathrm{e}$ perna $(5,5 \%)$. No tronco, as fraturas de costelas foram responsáveis por $8,5 \%$ do total e, na face, as de nariz alcançaram $4,9 \%$.

$\mathrm{Na}$ análise bruta, as quedas foram mais freqüentes no sexo feminino $(\mathrm{p}<0,001)$, nos mais velhos $(\mathrm{p}<0,001)$, nos idosos separados, divorciados e viúvos $(\mathrm{p}<0,001)$ e nos do nível socioeconômico mais baixo $(\mathrm{p}=0,04)$. O percentual de quedas esteve também associado com sedentarismo, autopercepção de saúde ruim e maior número de medicamentos referidos para uso contínuo. Em relação à utilização do serviço de atenção primária, os resultados mostram que não existe diferença entre a ocorrência de quedas nos idosos em função da modalidade de atenção da UBS. Na análise ajustada, as quedas em idosos se mantiveram associadas com sexo feminino, idade avançada, sedentarismo, autopercepção de saúde ruim, e maior número de medicamentos referidos para uso contínuo (Tabela 2).

A Tabela 3 apresenta a prevalência de quedas (intervalo de confiança de 95\%) nos subgrupos das variáveis independentes estratificado por sexo. Somente as variáveis com associação significativa na amostra total estão descritas na tabela 3. Em ambos os sexos, quanto maior a idade, maior a prevalência de quedas. $\mathrm{O}$ sedentarismo se associou positivamente com a ocorrência de quedas em ambos os sexos, embora a diferença entre ativos e sedentários tenha sido maior entre os homens. Os idosos que viviam separados ou divorciados, independentemente do sexo, foram os que apresentaram maior prevalência de quedas. Entre as mulheres, houve relação dose-resposta entre quedas e autopercepção de saúde. Já entre os homens, a tendência não foi clara, e somente o grupo que percebe a sua saúde como ruim foi estatisticamente diferente dos demais. Em ambos os sexos, houve relação direta
Tabela 1. Características da amostra segundo variáveis independentes. Estados do Sul e Nordeste do Brasil, 2005.

\begin{tabular}{|c|c|c|}
\hline Variável & $\mathrm{N}$ & $\%$ \\
\hline Sexo & 4.003 & \\
\hline Masculino & & 38,8 \\
\hline Feminino & & 61,2 \\
\hline Idade & 4.003 & \\
\hline 65 a 70 anos & & 38,5 \\
\hline 71 a 75 anos & & 25,7 \\
\hline 76 a 80 anos & & 18,5 \\
\hline Mais de 80 anos & & 17,3 \\
\hline Cor da pele & 4.003 & \\
\hline Branca & & 70,0 \\
\hline Não branca & & 30,0 \\
\hline Situação conjugal & 3.970 & \\
\hline Casado ou vive com companheiro & & 42,7 \\
\hline Viúvo & & 45,0 \\
\hline Separado ou divorciado & & 7,8 \\
\hline Solteiro & & 4,6 \\
\hline Situação de trabalho & 4.003 & \\
\hline Não aposentado & & 27,1 \\
\hline Aposentado & & 72,9 \\
\hline Escolaridade & 3.923 & \\
\hline Nenhuma & & 49,4 \\
\hline $1^{\circ}$ Grau incompleto & & 43,2 \\
\hline $1^{\circ}$ Grau completo $/ 2^{\circ}$ grau incompleto & & 3,9 \\
\hline $2^{\circ}$ Grau completo ou mais & & 3,5 \\
\hline Tabagismo & 4.000 & \\
\hline Não, nunca fumou & & 43,4 \\
\hline Já fumou & & 41,5 \\
\hline Fumante & & 15,2 \\
\hline Nível socioeconômico & 3.650 & \\
\hline A, B ou C & & 21,0 \\
\hline D & & 33,7 \\
\hline $\mathrm{E}$ & & 45,3 \\
\hline Sedentarismo & 3.944 & \\
\hline Não & & 42,1 \\
\hline Sim & & 57,9 \\
\hline Percepção da saúde & 3.951 & \\
\hline Excelente & & 2,6 \\
\hline Muito boa & & 3,2 \\
\hline Boa & & 29,7 \\
\hline Regular & & 43,8 \\
\hline Ruim & & 20,7 \\
\hline Tipo de UBS & 4.003 & \\
\hline Tradicional & & 34,5 \\
\hline PSF Pré-Proesf & & 36,0 \\
\hline PSF Pós-Proesf & & 29,6 \\
\hline Medicações referidas para uso contínuo & 4.003 & \\
\hline Nenhuma & & 31,6 \\
\hline 1 a 2 medicações & & 39,1 \\
\hline 3 a 4 medicações & & 21,3 \\
\hline 5 ou mais medicações & & 8,1 \\
\hline
\end{tabular}

UBS: Unidade Básica de Saúde

PSF: Programa Saúde da Família

Fonte: Projeto de Expansão e Consolidação da Saúde da Família (Proesf) 
Tabela 2. Prevalência de ocorrência de quedas em população idosa segundo variáveis independentes e análise bruta e ajustada. Estados do Sul e Nordeste do Brasil, 2005. N=4.003

\begin{tabular}{|c|c|c|c|c|c|}
\hline \multirow{2}{*}{ Variável } & \multicolumn{3}{|c|}{ Análise bruta } & \multicolumn{2}{|c|}{ Análise ajustada } \\
\hline & $\%$ & $\mathrm{RP}(\mathrm{IC} 95 \%)$ & $\mathrm{p}$ & RP (IC 95\%) & $\mathrm{p}$ \\
\hline Sexo & & & $<0,001$ & & $<0,001$ \\
\hline Masculino & 26,5 & 1 & & 1 & \\
\hline Feminino & 40,1 & $1,52(1,38 ; 1,67)$ & & $1,50(1,36 ; 1,66)$ & \\
\hline Idade & & & $<0,001$ & & $<0,001^{*}$ \\
\hline 65 a 70 anos & 31,8 & 1 & & 1 & \\
\hline 71 a 75 anos & 33,2 & $1,05(0,93 ; 1,17)$ & & $1,04(0,93 ; 1,17)$ & \\
\hline 76 a 80 anos & 36,8 & $1,16(1,03 ; 1,31)$ & & $1,14(1,01 ; 1,29)$ & \\
\hline Mais de 80 anos & 42,0 & $1,32(1,18 ; 1,48)$ & & $1,29(1,15 ; 1,45)$ & \\
\hline Cor da pele & & & 0,71 & & 0,68 \\
\hline Branca & 35 & 1 & & 1 & \\
\hline Não branca & 34,4 & $0,98(0,90 ; 1,08)$ & & $0,98(0,88 ; 1,08)$ & \\
\hline Situação conjugal & & & $<0,001$ & & 0,05 \\
\hline Casado ou vive com companheiro & 29,6 & 1 & & 1 & \\
\hline Viúvo & 38,8 & $1,31(1,19 ; 1,44)$ & & $1,04(0,94 ; 1,17)$ & \\
\hline Separado e divorciado & 39,8 & $1,35(1,15 ; 1,57)$ & & $1,23(1,05 ; 1,44)$ & \\
\hline Solteiro & 32,6 & $1,10(0,88 ; 1,38)$ & & $0,94(0,75 ; 1,17)$ & \\
\hline Situação de trabalho & & & 0,015 & & 0,74 \\
\hline Não aposentado & 37,8 & 1 & & 1 & \\
\hline Aposentado & 33,7 & $0,89(0,81 ; 0,98)$ & & $1,02(0,92 ; 1,13)$ & \\
\hline Escolaridade & & & 0,005 & & $0,15^{*}$ \\
\hline Nenhuma & 36,3 & 1 & & 1 & \\
\hline $1^{\circ} \mathrm{Grau}$ incompleto & 33,8 & $0,93(0,85 ; 1,02)$ & & $0,97(0,88 ; 1,06)$ & \\
\hline $1^{\circ}$ Grau completo $/ 2^{\circ}$ incompleto & 28,6 & $0,78(0,61 ; 1,01)$ & & $0,88(0,68 ; 1,14)$ & \\
\hline $2^{\circ}$ Grau completo ou mais & 27,2 & $0,75(0,57 ; 0,99)$ & & $0,85(0,64 ; 1,13)$ & \\
\hline Tabagismo & & & $<0,001$ & & 0,52 \\
\hline Nunca fumou & 37,8 & 1 & & 1 & \\
\hline Já fumou & 33,3 & $0,88(0,80 ; 0,97)$ & & $0,99(0,90 ; 1,08)$ & \\
\hline Fumante & 30,6 & $0,81(0,71 ; 0,93)$ & & $0,92(0,80 ; 1,06)$ & \\
\hline Nível socioeconômico & & & 0,04 & & $0,33^{*}$ \\
\hline A, B ou $C$ & 32,4 & 1 & & 1 & \\
\hline $\mathrm{D}$ & 33,5 & $1,04(0,91 ; 1,18)$ & & $1,00(0,88 ; 1,15)$ & \\
\hline E & 36,4 & $1,13(0,99 ; 1,27)$ & & $1,06(0,93 ; 1,21)$ & \\
\hline Sedentarismo & & & $<0,001$ & & $<0,001$ \\
\hline Não & 30,1 & 1 & & 1 & \\
\hline Sim & 38,3 & $1,28(1,17 ; 1,40)$ & & $1,20(1,09 ; 1,32)$ & \\
\hline Percepção da saúde & & & $<0,001$ & & $<0,001^{*}$ \\
\hline Excelente & 26,9 & 1 & & 1 & \\
\hline Muito boa & 25,6 & $0,95(0,62 ; 1,47)$ & & $0,89(0,58 ; 1,38)$ & \\
\hline Boa & 28,2 & $1,05(0,75 ; 1,46)$ & & $0,96(0,69 ; 1,33)$ & \\
\hline Regular & 35,4 & $1,32(0,95 ; 1,82)$ & & $1,14(0,83 ; 1,58)$ & \\
\hline Ruim & 45,5 & $1,67(1,22 ; 2,34)$ & & $1,39(1,00 ; 1,92)$ & \\
\hline Tipo de UBS & & & 0,98 & & 0,94 \\
\hline Tradicional & 34,6 & 1 & & 1 & \\
\hline PSF Pré-Proesf & 35,3 & $1,01(0,92 ; 1,13)$ & & $0,99(0,89 ; 1,10)$ & \\
\hline PSF Pós-Proesf & 34,6 & $1,00(0,90 ; 1,11)$ & & $1,00(0,91 ; 1,12)$ & \\
\hline Medicações referidas para uso contínuo & & & $<0,001$ & & $<0,001^{*}$ \\
\hline Nenhuma & 30,2 & 1 & & 1 & \\
\hline 1 a 2 medicações & 33,2 & $1,10(0,98 ; 1,22)$ & & $0,99(0,89 ; 1,11)$ & \\
\hline 3 a 4 medicações & 40,2 & $1,33(1,18 ; 1,50)$ & & $1,17(1,04 ; 1,33)$ & \\
\hline 5 ou mais medicações & 46,8 & $1,55(1,34 ; 1,78)$ & & $1,31(1,13 ; 1,53)$ & \\
\hline
\end{tabular}

* Valor de p para tendência

Fonte: Projeto de Expansão e Consolidação da Saúde da Família (Proesf) 
entre o número de medicamentos referidos para o uso contínuo e a ocorrência de quedas.

\section{DISCUSSÃO}

A prevalência de quedas encontrada foi de $34,8 \%$ em amostra de idosos residentes na área de abrangência de UBS. O resultado do presente estudo foi ligeiramente mais elevado do que outros encontrados na literatura internacional. Estudos relatam prevalência de quedas em idosos de $31,9 \%{ }^{8}$ e $28,5 \%{ }^{12}$ na Turquia, e na Cataluña, a prevalência de quedas que ocasionaram alguma restrição nas atividades habituais foi de $17,9 \%$. O presente estudo incluiu todas as quedas ocorridas nos últimos 12 meses, portanto não pode ser diretamente comparável ao resultado da Cataluña, que incluiu somente aquelas que determinaram alteração nas atividades habituais. ${ }^{21}$ No entanto os dados do presente estudo podem ser comparados a outro estudo realizado no Brasil, onde cerca de $31 \%$ dos idosos sofreram queda no ano anterior à entrevista. ${ }^{15}$
A diferença por sexo encontrada está de acordo com os relatos na literatura, com prevalência de quedas de $26,5 \%$ nos homens e 40,1\% nas mulheres. Esse valor é semelhante ao $43 \%$ encontrado em outro estudo realizado no País, de quedas no ano anterior a entrevista com mulheres que praticavam atividade física. ${ }^{11}$ Niino \& Kazokai, ${ }^{19}$ em um estudo no Japão, encontraram valores de $17,2 \%$ para mulheres e $8,3 \%$ nos homens, enquanto Assantachai et al $^{1}$ na Tailândia encontraram $24,1 \%$ e $12,1 \%$, respectivamente. Embora as prevalências tenham sido diferentes das encontradas em no presente estudo, a direção das associações foi consistente.

O número crescente de quedas com o aumento da idade é consistente com a literatura. ${ }^{3,4,13,19,21} \mathrm{O}$ envelhecimento traz perda de equilíbrio e alterações na massa muscular e óssea, aumentando as quedas. Uma das formas de minimizar essa perda decorrente do envelhecimento é a prática de atividades físicas. Dados atuais indicam que a prática de atividade física na adolescência e idade adulta diminui a ocorrência de quedas, osteoporose e outras doenças crônicas. ${ }^{22,23}$ No presente

Tabela 3. Prevalência e intervalos de confiança de variáveis associadas à queda de idosos nos últimos 12 meses, segundo sexo. Estados do Sul e Nordeste do Brasil, 2005.

\begin{tabular}{|c|c|c|c|c|c|c|}
\hline \multirow[t]{2}{*}{ Variável } & \multicolumn{2}{|c|}{ Homens } & \multicolumn{2}{|c|}{ Mulheres } & \multicolumn{2}{|r|}{ Total } \\
\hline & $\%$ & (IC 95\%) & $\%$ & (IC 95\%) & $\%$ & (IC 95\%) \\
\hline \multicolumn{7}{|l|}{ Idade } \\
\hline 65 a 70 anos & 23,9 & $(20,5 ; 27,3)$ & 36,8 & $(33,7 ; 39,9)$ & 31,8 & $(29,4 ; 34,1)$ \\
\hline 71 a 75 anos & 24,2 & $(19,9 ; 28,4)$ & 38,4 & $(35,0 ; 42,6)$ & 33,2 & $(30,3 ; 36,1)$ \\
\hline 76 a 80 anos & 25,4 & $(20,3 ; 30,5)$ & 44,2 & $(39,5 ; 48,8)$ & 36,8 & $(33,3 ; 40,3)$ \\
\hline Mais de 80 anos & 36,8 & $(31,0 ; 42,7)$ & 45,3 & $(40,5 ; 50,0)$ & 42,3 & $(38,3 ; 45,7)$ \\
\hline \multicolumn{7}{|l|}{ Sedentarismo } \\
\hline Não & 18,7 & $(15,6 ; 21,8)$ & 36,8 & $(33,8 ; 39,7)$ & 30,1 & $(27,8 ; 32,3)$ \\
\hline Sim & 31,7 & $(28,6 ; 34,7)$ & 42,7 & $(40,1 ; 45,3)$ & 38,3 & $(36,3 ; 40,3)$ \\
\hline \multicolumn{7}{|l|}{ Percepção da saúde } \\
\hline Excelente & 25,5 & $(13,6 ; 37,3)$ & 28,6 & $(15,5 ; 41,7)$ & 26,9 & $(18,3 ; 35,6)$ \\
\hline Muito boa & 18,3 & $(8,2 ; 28,4)$ & 32,3 & $(20,6 ; 44,0)$ & 25,6 & $(17,8 ; 33,4)$ \\
\hline Boa & 20,8 & $(17,3 ; 24,3)$ & 34,4 & $(30,7 ; 38,1)$ & 28,2 & $(25,7 ; 30,8)$ \\
\hline Regular & 27,1 & $(23,6 ; 30,6)$ & 40,1 & $(37,2 ; 42,3)$ & 35,4 & $(33,1 ; 37,7)$ \\
\hline Ruim & 38,0 & $(32,1 ; 43,9)$ & 49,0 & $(44,8 ; 53,2)$ & 45,5 & $(42,0 ; 48,9)$ \\
\hline \multicolumn{7}{|l|}{ Situação conjugal } \\
\hline Casado ou vive com companheiro & 25,0 & $(22,4 ; 27,5)$ & 38,4 & $(34,4 ; 42,3)$ & 29,6 & $(27,4 ; 31,8)$ \\
\hline Viúvo & 30,4 & $(25,1 ; 35,8)$ & 40,4 & $(37,9 ; 42,9)$ & 38,8 & $(36,5 ; 41,1)$ \\
\hline Separado e divorciado & 33,3 & $(24,0 ; 42,6)$ & 43,0 & $(36,2 ; 49,8)$ & 39,8 & $(34,3 ; 45,2)$ \\
\hline Solteiro & 26,2 & $(12,3 ; 40,0)$ & 34,5 & $(26,5 ; 42,5)$ & 32,6 & $(25,7 ; 39,4)$ \\
\hline \multicolumn{7}{|l|}{ Medicações referidas para uso contínuo } \\
\hline Nenhuma & 23,3 & $(20,0 ; 26,6)$ & 37,1 & $(33,3 ; 40,8)$ & 30,3 & $(27,1 ; 32,8)$ \\
\hline 1 a 2 medicações & 24,5 & $(20,9 ; 28,0)$ & 38,2 & $(35,1 ; 41,2)$ & 33,1 & $(30,8 ; 35,6)$ \\
\hline 3 a 4 medicações & 34,9 & $(29,0 ; 40,7)$ & 42,6 & $(38,6 ; 46,6)$ & 40,2 & $(37,0 ; 43,5)$ \\
\hline $5 \mathrm{ou}+$ medicações & 37,0 & $(26,9 ; 47,0)$ & 50,7 & $(44,1 ; 57,1)$ & 46,8 & $(41,3 ; 52,2)$ \\
\hline
\end{tabular}


estudo, idosos sedentários apresentaram uma maior prevalência de quedas, embora o delineamento transversal não permita a confirmação de causalidade nessa associação.

Estudos anteriores ${ }^{3,8,24}$ mostraram que a utilização de medicamentos aumenta a ocorrência de quedas. É necessário lembrar que os idosos que utilizam mais medicamentos normalmente são aqueles que realmente mais precisam e, consequentemente, estão submetidos a maior possibilidade de quedas. As conclusões desses estudos na literatura indicam a necessidade de uma revisão medicamentosa no sentido de prevenir a ocorrência de quedas.

Similarmente a estudo realizado em Barcelona, ${ }^{18} \mathrm{o}$ presente estudo encontrou que idosos separados ou divorciados apresentam elevada possibilidade de quedas. $\mathrm{O}$ cuidado mútuo entre parceiros pode explicar a ocorrência reduzida de quedas entre aqueles que vivem com companheiro. Melhorias na infra-estrutura dos domicílios devem ser realizadas no sentido de minimizar a ocorrência deste desfecho entre idosos.

$\mathrm{Na}$ amostra estudada, $12 \%$ das quedas resultaram em algum tipo de fratura, valor similar aos encontrados na literatura. Estudo no Japão ${ }^{14}$ encontrou prevalência de $10 \%$ de fraturas e de $17 \%$ em um estudo na Bélgica entre os indivíduos que sofreram queda.

Em relação à utilização dos serviços de saúde e a ocorrência de quedas, verificou-se que não há diferença entre os que utilizam os modelos PSF ou tradicional da atenção básica. Esforços devem ser realizados para prevenir a ocorrência de quedas. As elevadas prevalências encontradas no presente estudo sugerem que a atenção básica brasileira deve ser qualificada e reorganizada para atender os idosos, visando à prevenção da ocorrência deste importante agravo neste segmento da população. 


\section{REFERÊNCIAS}

1. Assantachaii P, Pradistsuwan R, Chatlhanawaree W, Pisalsarakij D, Thamlikitkul V. Risk factors for falls in the Tai elderly in an urban community. J Med Assoc Thai. 2003;86(2): 124-30.

2. Barros AJ, Hirakata VN. Alternatives for logistic regression in cross-sectional studies: an empirical comparison of models that directly estimate the prevalence ratio. BMC Med Res Methodol. 2003;3:21.

3. Blake AJ, Morgan K, Bendall MJ, Dallosso H, Ebrahim $\mathrm{SB}$, Arie TH, et al. Falls by elderly people at home: prevalence and associated factors. Age Ageing. 1988; 17(6):365-72.

4. Bueno-Cavanillas A, Padilla-Ruiz F, Jimenez-Moleon JJ, Peinado-Alonso CA, Galvez-Vargas R. Risk factors in fall among the elderly according to extrinsic and intrinsic precipitating causes. Eur J Epidemiol. 2000;16(9):849-59.

5. Craig CL, Marshall AL, Sjöström M, Bauman AE, Booth $M L$, Ainsworth BE, et al. International physical activity questionnaire: 12 -country reliability and validity. Med Sci Sports Exerc. 2003;35(8):1381-95.

6. Cummings SR, Melton LJ. Epidemiology and outcomes of osteoporotic fractures. Lancet. 2002;359(9319):1761-7.

7. Das CP, Joseph S. Falls in elderly. J Indian Med Assoc. 2005;103(3):136,138,140.

8. Evci ED, Ergin F, Beser E. Home accidents in the elderly in Turkey. Tohoku / Exp Med. 2006;209(4):291-301.

9. Fabrício SC, Rodrigues RA, Costa Junior ML. Falls among older adults seen at a Sao Paulo State public hospital: causes and consequences. Rev Saude Publica. 2004;38(1):93-9.

10. Facchini LA, Piccini RX, Tomasi E, Silveira DS, Siqueira VF, Rodrigues MA. Desempenho do PSF no sul e no nordeste do Brasil: avaliação institucional e epidemiológica da atenção básica à saúde. Cienc Saude Coletiva. 2006;11(3):669-81.

11. Guimarães JMN, Farinatti PTV Análise descritiva de variáveis teoricamente associadas ao risco de quedas em mulheres idosas. Rev Bras Med Esporte. 2005;11(5):299-305.

12. Hallil M, Ulger Z, Cankurtaran M, Shorbagi A, Yavuz $\mathrm{BB}$, Dede D, et al. Falls and the elderly: is there any difference in the developing world? A crosssectional study from Turkey. Arch Gerontol Geriatr. 2006;43(3):351-9.
13. Lord SR, Menz HB, Sherrington C. Home environment risk factors for falls in older people and the efficacy of home modifications. Age Ageing. 2006;35(Suppl 2): ii55-ii59.

14. Milisen K, Detroch E, Bellens K, Braes T, Dierickx K, Smeulders W, et al. Falls among community-dwelling elderly: a pilot study of prevalence circumstances and consequences in Flanders. Tijdschr Gerontol Geriatr. 2004;35(1):15-20.

15. Perracini MC, Ramos LR. Fatores associados a quedas em uma coorte de idosos residentes na comunidade. Rev Saude Publica. 2002;36(6):709-16.

16. Piccini RX, Facchini LA, Tomasi, E, Thumé E, Silveira DS, Siqueira FV, et al. Necessidades de saúde comuns aos idosos: efetividade na oferta e utilização em atenção básica à saúde. Cienc Saude Coletiva. 2006;11(3):657-67.

17. Monteiro CA. Novos e velhos males da saúde no Brasil: A evolução do país e de suas doenças. São Paulo: HUCITEC/USP; 1995.

18. Moreno-Martínez NR, Ruíz-Hidalgo D, BurdoyJoaquim E, Vázquez-Mata G. Incidência y factores explicativos de lãs caídas em ancianos que vivem em la comunidad. Rev Española de Geriatria y Gerontología. 2005;40(suppl 2):11-7.

19. Niino N, Kozakai R, Eto M. [Epidemiology of falls among community-dwelling elderly people]. Nippon Ronen Igakkai Zashi. 2003;40(5):484-6.

20. Rubenstein LZ. Falls in older people: epidemiology, risk factors and strategies for prevention. Age Ageing. 2006;35(Suppl 2):ii37-41.

21. Seculi Sanchez E, Brugulat Guiteras P, March Llanes J, Medina Bustos A, Martinez Beneyto V, Tressera Gaju R. [Falls in the elderly: knowing to act]. Aten Primaria. 2004;34(4):186-91.

22. Shepard RJ. Physical activity, fitness and health: the current consensus. Quest. 1995;47(3):228-303.

23. U.S. Department of Health and Human Services. Physical activity and health: a report of the surgeon general. Atlanta, GA; 1996.

24. Ziere G, Dieleman JP, Hofman A, Pols HA, Cammen TJ, Strieker BH. Polypharmacy and falls in the middle age and elderly population. Br J Clin Pharmacol. 2006;21(2):218-23. 